\title{
Redes sociales y aprendizaje cooperativo en un MOOC $^{1}$ \\ Social networks and cooperative learning in a MOOC

\author{
Carlos CASTAÑO GARRIDO, Inmaculada MAIZ OLAZABALAGA
} \\ y Urtza GARAY RUIZ \\ Universidad del País Vasco/Euskal Herriko Unibertsitatea
}

Recibido: Julio 2014

Aceptado: Noviembre 2014

\section{Resumen}

Los cursos MOOC están alterando el panorama educativo y recibiendo gran atención de la literatura científica. Sin embargo, el diseño pedagógico de estas propuestas ha sido puesto en entredicho. Es precisamente el aspecto social de los MOOC, esto es, la interacción entre los participantes del curso y el apoyo a los procesos de aprendizaje uno de los grandes temas de interés. En este artículo se presentan los resultados de una investigación realizada en la Universidad del País Vasco centrada en el aprendizaje cooperativo y en la utilización intensiva de Redes Sociales en un curso MOOC. Se han recopilado datos significativos mediante encuestas tipo Likert que revelan que el uso de las redes sociales, tanto externas como internas, de un curso online masivo y abierto, es un factor valorado positivamente por los estudiantes. Se defiende que la utilización de Redes Sociales como estrategia de aprendizaje en un curso MOOC influye en el rendimiento académico y las tasas de éxito de los estudiantes. Además, la edad de los participantes influye en las Redes Sociales utilizadas, encontrando que mientras que los más jóvenes tienden a trabajar en redes externas como Twitter o blogs personales, los de más edad se decantan por los foros que la plataforma Chamilo o la red Ning proporcionan.

Palabras clave: rendimiento, aprendizaje cooperativo, innovación educativa, educación superior, redes sociales.

\begin{abstract}
MOOCs are changing the educational landscape and gaining a lot of attention in scientific literature. However, the pedagogical design of these proposals has been called into question. It is precisely MOOCs' social aspect, i.e. the interaction between course participants and the support for learning processes that has become one of the main topics of interest. This article presents the results of a research project carried out at the University of the Basque Country, which focused in cooperative learning and the intensive use of social networks in a MOOC. Significant data was compiled through Likert-type surveys, revealing that the use of both external and internal social networks in a massive open online course is a factor that is evaluated positively by students. We argue that the use of social networks as a learning strategy

${ }^{1}$ Proyecto de investigación financiado por la UPV/EHU, con referencia EHU 13/59 (2013).


in a MOOC has an influence on academic performance and on the students' success rate. Furthermore, the participants' age also has a bearing on the social networks they use, and we have found that the younger members tend to work with external networks such as Twitter or personal blogs, whereas the older students are more inclined to use forums from the Chamilo or Ning platforms.

Keywords: learning success, cooperative learning, educational innovation, higher education, social networks.

Desde su aparición en 2008, los denominados MOOC están recibiendo gran atención en la literatura científica, presentando una nueva manera de enfocar la formación que está atrayendo a millones de alumnos en todo el mundo. De tal forma, que está afectando a la manera en que las universidades presenciales conciben la formación online.

Es interesante, además, atender a la inusitada rapidez con la que esta nueva tecnología disruptiva está encontrando eco en la educación (Castaño y Cabero, 2013; Haggard, 2013; Conole, 2014). Sirva como ejemplo el Informe Horizon de 2013 (Johnson et al., 2013, pp. 11-15), que señala los cursos online masivos y abiertos como una de las tendencias emergentes en la educación superior con un horizonte de implantación de un año o menor, aun cuando en el informe de 2012 (Johnson, Adams y Cummins, 2012) no encontrábamos ninguna referencia a este fenómeno.

La mayoría de las universidades están pendientes de esta nueva manera de enfocar la formación online, atendiendo al impacto que este tipo de cursos gratuitos podría tener sobre las ofertas educativas tradicionales. Esta ola disruptiva afectó a las universidades en el año 2012, de manera que, al final de ese año, 18 de las 20 mejores universidades de América del Norte ofrecían MOOC. Su aumento ha sido exponencial desde entonces. Europa en general y España en particular no es ajena a este fenómeno tal como se refleja en diferentes informes (Oliver et al, 2014; Open Education Europa, 2014), donde observamos que a fecha de 1 de julio de 2014, de una oferta europea, de un total de 673 cursos MOOC, 240 se ofertan en universidades españolas (35\%) (Open Education Europa).

Este interés académico se traslada también al mundo de la investigación. De esta manera, la Fundación Bill y Melinda Gates pone en marcha la Iniciativa de Investigación en MOOC (Gates Foundation, 2013) centrada en la evaluación de los MOOC y su impacto en la enseñanza, el aprendizaje y la educación en general. Por otra parte, comienzan a publicarse artículos que recogen el cuerpo de investigación en este tema (Liyanagunawardena, Adams y Williams, 2013; Castaño, 2013; Karsenti, 2013).

En todos los casos, la investigación relacionada con cuestiones pedagógicas es una constante en los estudios sobre MOOC, que con frecuencia son atacados de falta de rigor educativo (Vardi, 2012; Zapata-Ros, 2013; Guàrdia, Maina y Sangrà, 2013; Valverde, 2014). A pesar de estas críticas, y de que los MOOC utilicen un tipo de enseñanza flexible que tienen una estandarización pequeña (Shirky, 2013), diferentes autores proponen distinguir entre c-MOOC “conectivistas" versus X-MOOC más 
“tradicionales” (Siemens, 2012; Rodriguez, 2013), por lo que no cabría hablar de un único diseño pedagógico (Cabero, Llorente y Vázquez, 2014).

Así, Rodriguez (2012) estudia con detalle los cursos representativos de Inteligencia Artificial (AI) de Stanford (Udacity, Coursera) y los formatos c-MOOC, estableciendo que, a pesar de que ambos comparten el uso de las redes distribuidas, el formato asociado con c-MOOC, que se definen por un modelo pedagógico participativo, son únicos y diferentes del curso AI. Esta misma discusión es recogida por Bates (2012), quien critica que los métodos de enseñanza utilizados por la mayoría de los cursos de la plataforma Coursera hasta el momento, se basan en una pedagogía conductista muy anticuada y desactualizada.

Esta primera clasificación, demasiado simplista, va adquiriendo complejidad. Así, Knox, et al. (2012) buscan en el diseño de un curso MOOC desarrollado por la Universidad de Edimburgo superar estas deficiencias añadiendo a su curso la utilización de algunas de las prácticas más interesantes e innovadoras del e-learning. Entre ellas, evitar la "automatización” del curso, prestando atención al contacto y al diálogo entre alumnado y profesorado y entre los propios estudiantes, y poner en primer plano, más que la entrega de contenidos, las redes sociales (network) del proceso, la comunidad y el aprendizaje.

Este desarrollo teórico presentado por Knox et al. (2012) está en sintonía con la propuesta de Luisa Lane (2012) quien encuentra dificultades para situar su propuesta (POT Certificate Class, 2012) en la disyuntiva "Modelo Stanford" versus MOOC "conectivista", proponiendo un proyecto basado en tareas, al que denomina s-MOOC (Skill MOOC). Desde esta perspectiva de complejización (Conole, 2013), los cursos MOOC cooperativos intentan responder a la heterogeneidad de los participantes en un MOOC realizando un curso tipo $\mathrm{X}$, pero integrando algunas de las ventajas de los cursos conectivistas (Fidalgo, Sein-Echaluze y García Peñalvo, 2013): utilización intensiva de las redes sociales, creación de comunidades de aprendizaje (Alario-Hoyos et al., 2013) y utilización de entornos personales de aprendizaje (Castaño y Cabero, 2013). De esta manera, la interacción entre los participantes en un curso MOOC (alumnos y profesores), esto es, el aspecto social de los MOOC, se está convirtiendo en uno de los grandes temas de preocupación.

Efectivamente, como señalan Fidalgo et al., (2013), la incorporación de las redes sociales en los denominados xMOOC como parte de la estrategia de aprendizaje, permite dotar a éstos de las principales ventajas de los cMOOC. La cooperación en la generación de conocimiento se vuelve fundamental en este aspecto. No es mucha la investigación en este aspecto. Diferentes autores (Khalil y Ebner, 2013; Kolowich, 2013) indican que los MOOC hacen difícil, si no imposible, la interacción entre estudiantes y docentes, especialmente en los cursos con mayor número de participantes. Si bien otros estudios afirman que los MOOC ofrecen a los alumnos la oportunidad de interaccionar entre ellos, especialmente en los foros de discusión (Yeager, Hurley-Dasgupta y Bliss, 2013), parece cierto que la mayoría de estos no participa en estos foros (Kop, 2011; Kop, Fournier y Mak, 2011). Quizá, como opina Karsenti (2013), uno de los mayores desafíos de los MOOC es asegurar el apoyo al proceso de aprendizaje. Un proceso que requiere de alumnos autónomos. 
Siemens (2014) aborda esta cuestión afirmando que ambos tipos de cursos (xMOOC y cMOOC) son incompatibles, imposibles de mezclar, puesto que desde el punto de vista pedagógico y filosófico son demasiado diferentes. Sin embargo, plantea la posibilidad de diseñar un MOOC de dos capas de tal modo que a lo largo del curso el alumno pueda elegir una u otra (xMOOC o cMOOC) a su conveniencia. En este sentido, diversos estudios se están interesando por perfiles diferentes de alumnos, estudiando su motivación, perfil de compromiso y resultados de aprendizaje y tasas de finalización (Liyanagunawardena, 2014; Saadatmand y Kunpulainen, 2014; Poellhuber et al., Roy, Bouchoucha y Anderson, 2014; Wang y Baker, 2014). Por otra parte, diversos autores también recogen la importancia de seguir investigando sobre la interacción entre la utilización de redes sociales y rendimiento (Cheng, 2014; Eynon, 2014).

\section{Descripción y metodología}

El objetivo de esta investigación es analizar el uso de las redes sociales y el aprendizaje de los participantes en un curso MOOC cooperativo, atendiendo a la participación, el rendimiento académico y la cooperación basada en la utilización de redes sociales.

En relación al diseño pedagógico del MOOC, una vez decidida la temática del curso sobre los entornos personales de aprendizaje (PLEs), los cursos online masivos y abiertos y los contenidos digitales, se realizó un estudio Delphi mediante una encuesta tipo Likert de cinco opciones, con el fin de que expertos en e-learning y TICs pertenecientes a universidades europeas y latinoamericanas dieran su opinión sobre el diseño del MOOC. Las preguntas que se hicieron versaban sobre el tipo de MOOC que parecía más apropiado, los materiales que se consideraban más acordes con el curso, el tipo de actividades a plantear, la importancia de la cooperación entre el alumnado y el profesorado. Tras el análisis de las 53 respuestas obtenidas, se diseñó un curso cuya principal característica era la cooperación, factor mejor valorado por los expertos en relación al aprendizaje en los MOOC. Posteriormente, el diseño pedagógico del MOOC se sometió a una segunda vuelta de recogida de opiniones de los expertos para dar por definitiva la estructura diseñada.

El curso se estructuró alrededor de seis temas con seis semanas de duración (uno por semana). Cada tema debía constar de al menos dos micropastillas de vídeo o polimedias, un documento en pdf no muy extenso (entre ocho y diez páginas), archivos de audio, materiales complementarios y de ampliación disponibles en la red y dos eactividades con sus correspondientes rúbricas de evaluación. 
El programa del curso quedó configurado de la siguiente forma (Tabla 1):

\begin{tabular}{l}
\hline TEMAS \\
\hline Tema 0.- Presentación \\
Tema 1.- Web 2.0 y educación \\
Tema 2.- El tsunami del Mobile Learning \\
Tema 3.- Recursos Educativos Abiertos (REA's) \\
Tema 4.- Entornos Personales de Aprendizaje (PLE’s) \\
Tema 5.- Enseñar en la sociedad del conocimiento: la evolución del e-learning \\
Tema 6.- El reto de los MOOC’s \\
\hline
\end{tabular}

Tabla 1. Programa del curso.

Se consideró necesario publicar una presentación del curso a través de micropastillas de vídeo en euskera y español (los idiomas oficiales del curso) para su difusión a través de Internet, junto con el programa y las preguntas más frecuentes. Además se puso a disposición de todas las personas interesadas un tutorial con las instrucciones para la inscripción en la plataforma del curso. Toda esta información pasó a constituir el tema 0 junto con la realización de una encuesta a los participantes para conocer algunas de sus características personales como la edad, sexo, estudios, experiencia con MOOC, con herramientas web y el grado de utilización de redes sociales.

La opción elegida para alojar el curso fue la plataforma Metauniversidad porque estaba basada en Chamilo, solución de software libre de gestión del e-learning y licenciada bajo la GNU/GPLx3. El profesorado implicado se había formado anteriormente para conocer las posibilidades que ofrecía Chamilo.

Una de las cuestiones más relevantes a la hora de ofrecer un curso online, masivo y abierto es la difusión del mismo para conseguir cumplir con la característica de masivo. En este caso se optó por darlo a conocer a través de la página Open Education Europa por la capacidad de distribución de información de la misma. Pero, por otra parte, era importante que se publicitara también bajo las siglas de la universidad que financiaba el proyecto, por lo que se optó por redirigir la información del curso al servicio de blogs de la UPV/EHU Ehusfera y desde este lugar se podía acceder al curso propiamente dicho.

El interés hacia un curso MOOC colaborativo basado en algunos aspectos del conectivismo, donde se primaban las conexiones y la cooperación entre los participantes, necesitaba una herramienta potente con el fin de favorecer las relaciones y las discusiones sobre los diferentes temas. Por lo que, además de los foros que la propia plataforma Chamilo presentaba, se abrió un canal en la red social Ning para favorecer el establecimiento de discusiones entre los inscritos en el curso y que además permitiera la apertura de distintos blogs dentro de la propia red.

Estos eran los elementos mínimos necesarios para comenzar un curso MOOC de las características descritas anteriormente, pero no se puede olvidar que las personas que participaron podían estar utilizando otras redes sociales como Twiter, Facebook, 
Linkedin, etc. y en ningún caso se quería impedir que continuaran haciéndolo, es más, se puso énfasis en que difundieran, a través de las distintas redes sociales, las eactividades que iban completando.

Finalmente la recogida de datos cuantitativos para su análisis posterior se realizó a través de varias fuentes: los personales estaban consignados en la encuesta 0 , anteriormente citada, y los de rendimiento derivaban de la evaluación de las nueve eactividades planteadas en la totalidad de los temas. También se administraron dos cuestionarios ya estandarizados (IMMS y TAM) que indagaban sobre las opiniones de los participantes sobre el diseño del MOOC. La escala IMMS (Instructional Materials Motivation Survey) constaba de 36 ítem que versaban en torno a la motivación, divididos en cuatro variables: atención, confianza satisfacción y relevancia. El cuestionario TAM (Technology Acceptance Model) se centraba en la percepción de la utilidad, la facilidad de uso y el disfrute en el desempeño unido al diseño pedagógico del curso desde las perspectiva de los participantes (Wojciechowski y Cellary, 2013). A todo ello, se sumaba un cuestionario específico sobre el nivel de uso de las redes sociales por los estudiantes antes y después de realizar el MOOC.

\section{Resultados.}

A continuación presentamos los resultados referentes a la investigación divididos en tres bloques para su mejor comprensión: la participación, el rendimiento logrado por los estudiantes y el análisis de la cooperación basada en la utilización de las redes sociales.

\section{Participación}

Podemos considerar que el MOOC obtuvo una participación activa alta teniendo en cuenta las tasas de abandono que se cifran en aproximadamente un 90\% (Álvarez, 2013; Jordan, 2013) en este tipo de cursos. En el inicio del curso se inscribieron setecientas cuarenta y cuatro personas.

Este número se redujo considerablemente en el tema introductorio o tema 0 donde se les pedía que completaran una serie de preguntas sobre sus datos y experiencias anteriores y ciento ochenta y seis (186) de la totalidad de los inscritos lo hicieron. De entre ellos ochenta y ocho participantes finalizaron las tareas de la primera semana de curso.

A continuación se recogen en la tabla 2 los datos porcentuales de participación:

\begin{tabular}{lr}
\hline Inscritos en el curso & $\mathbf{7 4 4}$ \\
Porcentaje de estudiantes que inician el curso (act.0) sobre el total de inscritos 186/744 & $25 \%$ \\
Porcentaje de estudiantes que finalizan sobre el total que inician (act.0) 69/186 & $37,09 \%$ \\
Porcentaje de estudiantes que realizan algunas de las actividades del curso sobre los que & $47,31 \%$ \\
inician (act.0) 88/186 & \\
Porcentaje de estudiantes que finalizan sobre los que realizan alguna de las actividades 69/88 & $78,40 \%$ \\
\hline
\end{tabular}

Tabla 2. Datos globales de participación en porcentajes. 
Otro dato sobre el distinto grado de participación de los sujetos que cursaron el MOOC es el que proporciona el número de personas que finalizaron cada una de las eactividades propuestas. Los resultados varían desde la actividad 0 hasta la novena, con ciento ochenta y seis (186) en el primer caso y sesenta y nueve (69) en el último, tal y como podemos observar en la tabla 3.

\begin{tabular}{lcc}
\hline $\begin{array}{l}\text { Número de estudiantes en cada } \\
\text { actividad }\end{array}$ & N & Porcentaje \\
\hline Actividad 0 & 186 & $100 \%$ \\
Actividad 1 de la semana 1 & 88 & $47,13 \%$ \\
Actividad 2 de la semana 1 & 88 & $47,13 \%$ \\
Actividad 3 de la semana 2 & 79 & $42,47 \%$ \\
Actividad 4 de la semana 2 & 79 & $42,47 \%$ \\
Actividad 5 de la semana 3 & 79 & $42,47 \%$ \\
Actividad 6 de la semana 4 & 71 & $38,17 \%$ \\
Actividad 7 de la semana 4 & 70 & $37,63 \%$ \\
Actividad 8 de la semana 5 & 70 & $37,73 \%$ \\
Actividad 9 de la semana 6 & 69 & $37,09 \%$ \\
\hline
\end{tabular}

Tabla 3. Participación en actividades por semanas.

\section{Rendimiento}

Los datos de rendimiento de los estudiantes, se obtuvieron a través de las notas con las que se evaluaron las nueve e-actividades propuestas. La nota media final resultante fue de 6,59 sobre 10, y en cada una de las e-actividades la nota media de todos los estudiantes estuvo por encima del 6,43, excepto en la actividad número nueve de la última semana de curso en que descendió a 5,69 puntos (ver tabla 4).

\begin{tabular}{|c|c|c|c|c|c|c|c|c|c|}
\hline \multicolumn{10}{|c|}{ Nota media por actividad } \\
\hline $\begin{array}{c}\text { Act. } \\
1\end{array}$ & $\begin{array}{c}\text { Act. } \\
2\end{array}$ & $\begin{array}{c}\text { Act. } \\
3\end{array}$ & $\begin{array}{c}\text { Act. } \\
4\end{array}$ & $\begin{array}{c}\text { Act. } \\
5\end{array}$ & $\begin{array}{c}\text { Act. } \\
6\end{array}$ & $\begin{array}{c}\text { Act. } \\
7\end{array}$ & $\begin{array}{c}\text { Act. } \\
8\end{array}$ & $\begin{array}{c}\text { Act. } \\
9\end{array}$ & $\begin{array}{c}\text { Nota media } \\
\text { global }\end{array}$ \\
\hline 6,68 & 6,85 & 6,44 & 7,17 & 6,44 & 7,15 & 7,27 & 7,29 & 5,69 & 6,59 \\
\hline
\end{tabular}

Tabla 4. Calificaciones por actividades.

Por otra parte, si las notas obtenidas en las e-actividades se consideran de manera global, la mayoría de los estudiantes consigue una calificación mayor de 7 puntos. En el gráfico 1 se recoge como el $12.68 \%$ de los participantes obtiene una calificación entre 5 y 6,99, el 76,05\% ha conseguido un notable y el 7,04 está por encima del 9. Solamente el $4,22 \%$ de los estudiantes no supera el curso. 


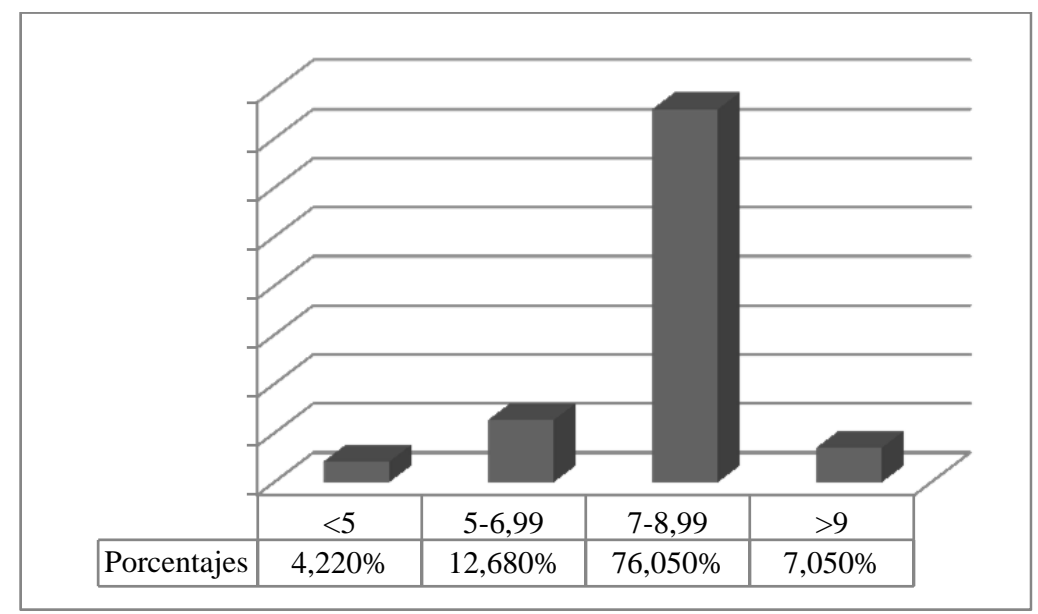

Gráfico 1. Calificaciones globales.

Si separamos por edades los datos de las calificaciones, son un $12 \%$ de los estudiantes mayores de 31 años los únicos que no llegan al aprobado. En el intervalo de notas de 5 a 6,99 encontramos al 8,45\% del grupo de menor edad y el 66,19\% en el intervalo del 7 al 8,99. Las notas más altas las consiguieron en el grupo de igual o menores de 31 años (ver tablas 4 y 5).

\begin{tabular}{lcc}
\hline & Frecuencia & Porcentaje \\
\hline $5-6.99$ & 6 & 10,7 \\
$7-8.99$ & 47 & 83,9 \\
$9-10$ & 3 & 5,4 \\
Total & 56 & 100,0 \\
\hline
\end{tabular}

Tabla $\overline{4 \text {. Calificaciones del grupo de edad de }}<=31$ años.

\begin{tabular}{rcc}
\hline & Frecuencia & Porcentaje \\
\hline$<5$ & 3 & 20,0 \\
$5-6.99$ & 3 & 20,0 \\
$7-8.99$ & 7 & 46,7 \\
$9-10$ & 2 & 13,3 \\
Total & 15 & 100,0 \\
\hline
\end{tabular}

Tabla 5. Calificaciones del grupo de edad de $>$ de 31 años.

Se podría pensar, por tanto, con los datos de participación y resultados obtenidos que el hecho de utilizar herramientas de cooperación, como las redes sociales en cursos MOOC, favorece el aprendizaje y la obtención de calificaciones positivas. 


\section{Cooperación en las redes sociales}

A lo largo del curso y en el desarrollo de las e-actividades propuestas, se insistió en la utilización de las redes sociales para conseguir que los estudiantes cooperaran entre ellos y también con los profesores del curso. El interés de los investigadores por observar cómo se tejían redes, se mantenían y se fortalecían llevó a incluir la creación de redes en las propias actividades planteadas, de manera que una de las exigencias era la de difundir sus trabajos a través de cualesquiera de las redes sociales que funcionan en Internet.

Para facilitar al alumnado estas tareas, como ya se ha dicho, se abrió un canal en la red social Ning donde se podían abrir discusiones e incluso hacer aportaciones en un blog dentro de la propia red. Pero, además los estudiantes podían aportar sus noticias y opiniones en las redes que habitualmente utilizaban como Twitter, Facebook, Linkedin, así como en sus blogs personales.

A continuación reseñamos en la tabla 6 el número de aportaciones, discusiones y entradas de blog que se recogieron entre los estudiantes y el profesorado del MOOC en la red social Ning:

\begin{tabular}{lc}
\hline Tipo de actuaciones & $\begin{array}{c}\text { Número de } \\
\text { actuaciones }\end{array}$ \\
\hline Participantes & 194 \\
Entradas & 772 \\
Discusiones o foros abiertos & 121 \\
Entradas en el blog & 49 \\
\hline
\end{tabular}

Tabla 6. Participaciones en la red social Ning.

En relación a las entradas realizadas en los blogs que los participantes ya tenían abiertos se contabilizaron cincuenta y siete (57) blogs. Los estudiantes reflejaron en sus bitácoras las distintas tareas que se les encargaron durante el curso, como por ejemplo la grabación de un podcast, la elaboración de blogsters con temáticas diferentes y las opiniones sobre tópicos planteados en alguno de los temas. Además también se contabilizaron setenta y cuatro (74) miembros activos en Twitter. En la tabla 7, se recogen estas aportaciones que se elaboraron con herramientas de cooperación disponibles en la red y elegidas por los propios estudiantes.

\begin{tabular}{ll}
\hline Aportaciones y herramientas & X \\
\hline Blogs & 57 \\
Miembros activos de Twiter & 74 \\
\hline
\end{tabular}

Tabla 7. Aportaciones y herramientas externas utilizadas. 
En cuanto a lo que se refiere a la utilización de las redes sociales por parte de los estudiantes en el desarrollo del MOOC, encontramos que el alumnado consideró, en general, que el MOOC le había proporcionado la oportunidad de utilizar las redes sociales para aprender. Así el 46,5\% considera que ha utilizado mucho las redes y el $25,4 \%$ bastante, frente a un $1,4 \%$ que señalaba que lo había hecho y un $8,5 \%$ que las había utilizado poco.

Los tipos de redes utilizadas por el alumnado durante el MOOC fueron Twitter, Facebook, foros, blogs, Youtube, Skype y Linkedin. En todos los casos el uso que hacían los estudiantes de estas redes antes del curso aumentó considerablemente después, como se puede apreciar en el gráfico 2:

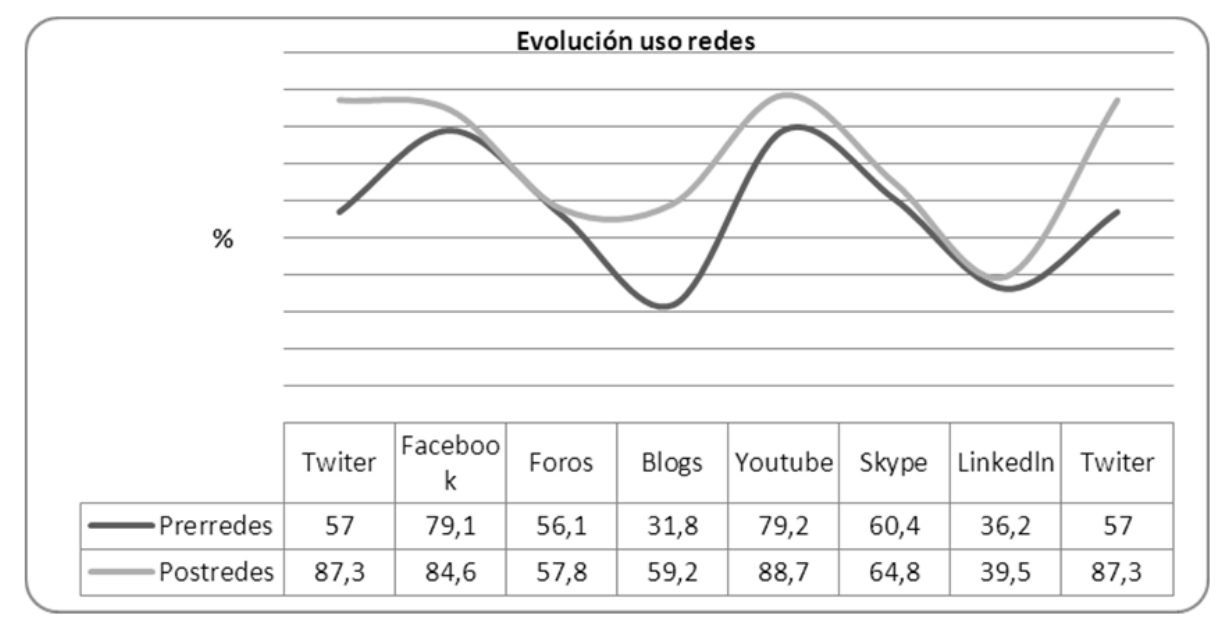

Gráfico 2. Uso de redes sociales antes y después del MOOC.

Entre las ya mencionadas redes encontramos que en el grupo de iguales o menores de 31 años usaron, de forma significativa, las siguientes herramientas: Twitter, Facebook, blogs y Linkedin. A diferencia de los mayores de 31 que se decantaron más por el uso de foros. 


\begin{tabular}{|c|c|c|c|c|c|c|c|c|c|}
\hline \multicolumn{10}{|c|}{ Diferencias relacionadas } \\
\hline & & \multirow[b]{2}{*}{ Media } & \multirow[b]{2}{*}{$\mathbf{S}$} & \multirow[b]{2}{*}{$\begin{array}{l}\text { Error tip. } \\
\text { de la media }\end{array}$} & \multicolumn{5}{|c|}{$\begin{array}{l}\text { 95\% intervalo de confianza para la } \\
\text { diferencia } \\
\end{array}$} \\
\hline & & & & & Inf & Sup & $\mathbf{T}$ & gl & $\begin{array}{c}\text { Sig. } \\
\text { (bilateral) }\end{array}$ \\
\hline Par 1 & $\begin{array}{l}\text { Val Exp Mooc - Val } \\
\text { Exp Моoc }\end{array}$ & $-3,170$ & 1,749 & ,255 & $-3,684$ & $-2,657$ & $-12,430$ & 46 & ,000 \\
\hline Par 2 & $\begin{array}{l}\text { t USO Redes - t USO } \\
\text { Redes }\end{array}$ &,- 347 & 1,347 & ,192 &,- 734 &, 040 & $-1,803$ & 48 & ,078 \\
\hline Par 3 & EXP Twi - EXP Twi & $-1,061$ & 1,391 & ,199 & $-1,461$ &,- 662 & $-5,342$ & 48 & ,000 \\
\hline Par 4 & EXP Face - EXP Face &,- 510 & 1,543 & ,220 &,- 953 &,- 067 & $-2,315$ & 48 & ,025 \\
\hline Par 5 & $\begin{array}{l}\text { EXP Foros - EXP } \\
\text { Foros }\end{array}$ &,- 816 & 1,395 & ,199 & $-1,217$ &,- 416 & $-4,098$ & 48 & ,000 \\
\hline Par 6 & EXP Marc - EXP Marc &,- 878 & 1,317 & ,188 & $-1,256$ &,- 499 & $-4,664$ & 48 & ,000 \\
\hline Par 7 & EXP You - EXP You &,- 224 & 1,311 & ,187 &,- 601 & ,152 & $-1,198$ & 48 & ,237 \\
\hline Par 8 & $\begin{array}{l}\text { EXP SKYPE - EXP } \\
\text { SKYPE }\end{array}$ &,- 327 & 1,360 & ,194 &,- 717 & ,064 & $-1,681$ & 48 & 099 \\
\hline Par 9 & $\begin{array}{l}\text { EXP LINKEDIN - } \\
\text { EXP LINKEDIN }\end{array}$ &,- 531 & 960 & 137 &,- 806 &,- 255 & $-3,870$ & 48 & ,000 \\
\hline
\end{tabular}

Tabla 8. Tipos de redes utilizadas

Siguiendo el análisis del uso de redes, ha quedado constatado que según el 59,2\% de los participantes el blog es la herramienta más útil para aprender interaccionando con otras personas. Además, encontramos también significatividad en el uso de esta herramienta (ver tabla 9), y en dos ítems donde se les preguntaba sobre la interacción con los compañeros vía red como potenciador del aprendizaje (DIS3) y si les parecía útil para el aprendizaje interaccionar con otras personas a través de redes sociales (PU7) tal y como podemos observar en la tabla 10. Estos ítems iban dirigidos hacía la experiencia con blogs en el MOOC.

\begin{tabular}{|c|c|c|c|}
\hline \multicolumn{2}{|c|}{ Variables de control } & AT 19 & \\
\hline AT 19 & $\begin{array}{l}\text { Correlación } \\
\text { Sig.(bilateral) } \\
\text { Gl }\end{array}$ & 1,000 & $\begin{array}{l}, 259 \\
\mathbf{0 7 9} \\
45 \\
\end{array}$ \\
\hline $\begin{array}{l}\text { EXP } \\
\text { Blogs }\end{array}$ & $\begin{array}{l}\text { Correlación } \\
\text { Sig.(bilateral) } \\
\text { gl }\end{array}$ & $\begin{array}{l}, 259 \\
, 079 \\
45\end{array}$ & 1,000 \\
\hline
\end{tabular}

Tabla 9. Correlaciones experiencia con Blogs en participantes $<=31$ años. 


\begin{tabular}{llllll}
\cline { 3 - 6 } & & DIS 3 & DIS 4 & PU 7 & $\begin{array}{c}\text { EXP } \\
\text { Blogs }\end{array}$ \\
\hline EXP & Correlación &, 349 &, 171 &, 296 & 1,000 \\
Blogs & $\begin{array}{l}\text { Sig.(bilateral) } \\
\text { gl }\end{array}$ &, 015 &, 244 &, 041 & \\
& 46 & 46 & 46 & 0 \\
\hline
\end{tabular}

Tabla 10. Correlaciones experiencia con Blogs, DIS 3 y PU 7.

Aunque los blogs hayan sido la herramienta más valorada para el aprendizaje mediante la interacción, la red social externa más utilizada fue Twitter, algo que se subraya de forma más significativa entre los participantes menores o iguales a 31 años (ver tabla 11). En general, más de la mitad de los participantes del MOOC, un 87,3\%, señaló haberla utilizado de forma constante durante el curso online, masivo y abierto realizado.

\begin{tabular}{llll}
\hline \multicolumn{2}{c}{ Variables de control } & AT 19 & EXP Twiter \\
\hline AT 19 & Correlación & 1,000 &, 299 \\
& Sig. (bilateral) & $\cdot$ & $\mathbf{, 0 4 1}$ \\
& gl & 0 & 45 \\
\hline EXP Twi & Correlación &, 299 & 1,000 \\
& Sig. (bilateral) &, 041 & $\cdot$ \\
& gl & 45 & 0 \\
\hline
\end{tabular}

Tabla 11. Correlaciones experiencia con Twiter en participantes $<=31$.

Otro de los análisis realizados fue el del uso de las redes dependiendo del tipo de estudiante que se consideraba cada participante: individualista o colaborador y activo, moderadamente activo u oculto. A pesar de que, a continuación, vamos a ir revisando los resultados con más detalle, en general podemos destacar que la percepción que el alumnado tenía de sí mismo cambia a lo largo del curso, coincidiendo con su consideración de que colaborar entre iguales resulta positivo para el aprendizaje. Es decir, los estudiantes se consideraron más activos y colaboradores de lo que pensaban ser al inicio del curso.

De este resultado podemos destacar que en lo que se refiere a autodefinirse como individualista o colaborativo se puede apreciar una diferencia significativa en relación a la categoría de estudiante individualista $(0,026)$. Es decir, que entre la encuesta realizada al comienzo del curso y la completada al final hay diferencias en el número de participantes que se consideran activos (Pre 1,76-Post 2,37). Por lo que se puede concluir, que al finalizar el curso el 57,8\% se definió poco individualista y el 84,6 se percibía como estudiante de tipo colaborador.

Esto es, que los participantes en el desarrollo del MOOC se fueron dando cuenta de que realmente tendían menos de lo que pensaban al trabajo individual. Son más 
colaboradores de lo que creían que iban a ser al inicio del curso y el tipo de participación fue más activa de lo que en un principio suponían. Es decir, al comenzar las actividades un 76,4\% consideraba que su participación iba a ser activa y al final fue un $82,2 \%$ el que se percibió como activo. En cambio, de los 76,4\% que creían que esta iba a ser oculta y 43,3\% que se definían como moderadamente ocultos, sólo lo fueron un $21,2 \%$ y un $38 \%$ respectivamente. Datos que coinciden con el punto de vista expresado por los participantes del MOOC que valoraban de forma significativa que ser un participante activo (DIS 3) y utilizar una red social como complemento del curso (DIS 4) e interaccionar mediante ellas contribuye a realizar mejor el MOOC (PU 7).

\begin{tabular}{cllllll}
\cline { 2 - 6 } & $\begin{array}{c}\text { Variables de } \\
\text { control }\end{array}$ & \multicolumn{1}{c}{ DIS 3 } & DIS 4 & \multicolumn{1}{c}{ PU 7 } & $\begin{array}{c}\text { PAR } \\
\text { ModOcul }\end{array}$ \\
\hline PAR & Correlación &,- 309 &,- 248 &,- 418 & 1,000 \\
ModOcul & $\begin{array}{l}\text { Sig.(bilateral) } \\
\text { gl }\end{array}$ &, 033 &, 090 &, 003 & \\
& 46 & 46 & 46 & 0 \\
\hline
\end{tabular}

Tabla 12. Correlaciones DIS 3, DIS 4, PU 7 y participante modo oculto.

Este último aspecto lo corroboran los alumnos que se describen como individualistas tal y como podemos observar a continuación (ver tabla 13):

\begin{tabular}{clllll}
\hline & & DIS 3 & DIS 4 & PU 7 & $\begin{array}{c}\text { EST } \\
\text { Indiv }\end{array}$ \\
\hline \multirow{2}{*}{ EST } & Correlación &,- 286 &,- 300 &,- 300 & 1,000 \\
Indiv & $\begin{array}{l}\text { Sig.(bilateral) } \\
\text { gl }\end{array}$ &, 049 & $\mathbf{, 0 3 9}$ & $\mathbf{, 0 3 8}$ & \\
& gl & 46 & 46 & 0 \\
\hline
\end{tabular}

Tabla 13. Correlaciones de estudiante individual con DIS 4 y PU 7 en el grupo de $<=31$ años.

Así, observamos que tanto en el ítem sobre la utilización de una red social como complemento del curso (DIS4), como en el que se refiere a la percepción de la utilidad para el aprendizaje interaccionando con otras personas a través de redes sociales (PU7) se extrae un resultado significativo: 0,039 y 0,038. Sobre este último ítem también se constata un resultado positivo entre los participantes menores o iguales a 31 años que se definen como implicados en el curso $(0,013)$.

Siguiendo con el uso de las herramientas, tanto los estudiantes menores o iguales a 31 años que se auto-describieron como individualistas $(0,001)$ y colaboradores $(0,001)$ e implicados $(0,060)$, como aquellos participantes mayores de 31 años que se autodefinieron como individualistas $(0,000)$ y colaboradores $(0,098)$ e implicados $(0,067)$ consideraron que utilizar una red social en el curso, como ha sido NING, contribuye a realizarlo con mayor facilidad.

Por el contrario, no hubo consenso entre los estudiantes de las diferentes franjas de 
edad sobre las herramientas mejor valoradas para la interacción. Mientras que para el grupo de participantes menores o iguales a 31, y coincidiendo con los resultados generales presentados anteriormente, Twitter $(0,041)$ y los blogs $(0,079)$ son las redes que más se utilizan y que más aportan a la interacción; en el caso de los mayores de 31 años la significatividad la encontramos, de nuevo, en la utilización y validez de los foros $(0,075)$ para el aprendizaje basado en la interacción.

Desde esta perspectiva, en lo que se refiere a la percepción que el alumnado tenía sobre la importancia de la interacción con los demás para el propio aprendizaje, el resultado es similar para ambos grupos de edad. La diferencia se encuentra en que mientras que los mayores de 31 años la consideraban importante, los menores o iguales a 31 años dieron un paso más y expresaron que la interacción no sólo es importante para el aprendizaje sino que lo potencia como se puede apreciar en las correlaciones de las tablas 14, 15 y 16.

\begin{tabular}{cllll}
\hline Variables de control & DIS 4 & PU 7 & $\begin{array}{c}\text { PAR } \\
\text { ModOcul }\end{array}$ \\
\hline \multirow{2}{*}{ DIS 3 } & Correlación &, 576 &, 693 &,- 309 \\
\cline { 2 - 5 } & Sig.(bilateral) &, 000 &, 000 & $\mathbf{, 0 3 3}$ \\
\cline { 2 - 5 } & gl & 46 & 46 & 46 \\
\hline
\end{tabular}

Tabla 14. Correlaciones participante moderadamente oculto con DIS 3 en $<=31$ años.

\begin{tabular}{cllll}
\hline Variables de control & DIS 4 & PU 7 & $\begin{array}{c}\text { EST } \\
\text { Indiv }\end{array}$ \\
\hline \multirow{2}{*}{ DIS 3 } & Correlación &, 547 &, 668 &,- 286 \\
\cline { 2 - 5 } & Sig.(bilateral) &, 000 &, 000 &, 049 \\
\cline { 2 - 5 } & gl & 46 & 46 & 46 \\
\hline
\end{tabular}

Tabla 15. Correlaciones estudiante individual con DIS 3 en $<=31$ años.

\begin{tabular}{lllll}
\hline Variables de control & DIS 4 & PU 7 & $\begin{array}{c}\text { IMP } \\
\text { Impli }\end{array}$ \\
\hline \multirow{2}{*}{ DIS 3} & Correlación &, 535 &, 658 &, 349 \\
\cline { 2 - 5 } & Sig.(bilateral) &, 000 &, 000 & $\mathbf{, 0 1 5}$ \\
\cline { 2 - 5 } & gl & 46 & 46 & 46 \\
\hline
\end{tabular}

Tabla 16. Correlaciones estudiante implicado con DIS 3 en $<=31$ años.

En cambio, curiosamente, es entre los participantes mayores de 31 años que se describen como ocultos donde encontramos significatividad (ver tabla 16). Esto es, a pesar de considerar importante implicarse en las actividades interactivas para el aprendizaje personal, parece que ellos no se involucraron demasiado. 


\begin{tabular}{cll}
\hline & & \multicolumn{1}{c}{ PAR } \\
Variables de control & Oculto \\
\hline & Correlación &,- 700 \\
AT 19 & Sig.(bilateral) & $\mathbf{, 0 1 1}$ \\
& gl & 10 \\
\hline
\end{tabular}

Tabla 17. Correlaciones participante oculto e interacción en <= 31 años.

Debemos añadir que este resultado positivo se dio en los dos grupos de edad tanto en los estudiantes individualistas como colaborativos e implicados, que mayoritariamente señalaron que la interacción con los compañeros a través de las redes les ayudó en el desarrollo del curso. Así, el 94,1\%, frente al 5,9\%, expresó su acuerdo con que la interacción con los compañeros vía red potencia el aprendizaje; el 87,1\% frente al $12,9 \%$ que utilizar, por lo menos, una red social como complemento del MOOC le había ayudado a realizarlo, y finalmente, al 96,5 frente al 3,6 le pareció útil para el aprendizaje interaccionar con otras personas a través de redes sociales.

En resumen, al 78,9\% de los estudiantes de todas las edades le pareció importante la interacción con los compañeros a través de Internet para mantener la atención sobre el curso, aunque su participación haya sido de distinto tipo: individualista, colaborativo o implicado en el curso.

\section{Discusión de resultados y conclusiones}

La utilización intensiva de Redes Sociales en el desarrollo de un MOOC, ha sido positivamente valorada por los participantes, habiendo encontrado además significatividad entre la interacción con los compañeros y la utilidad de la red como potenciadora del aprendizaje. Es necesario señalar, sin embargo, que las plataformas convencionales utilizadas para desarrollar cursos MOOC no cuentan con herramientas potentes para facilitar esta interacción, más allá de los foros. Esto hace necesaria la utilización de herramientas externas a las propias plataformas,. En nuestro caso una red Social, Ning, y la utilización de las propias herramientas por parte de los participantes (blogs, Twitter, Facebook, etc.).

Según los resultados de este estudio la dificultad viene dada en ver cómo y qué tipo de redes utilizan, ya que hemos podido comprobar que la edad influye en la elección de herramientas sociales externas o internas al curso. Así, tal y como se ha señalado, son los participantes más jóvenes los que tienden a redes externas como Twitter o blogs personales, mientras que los mayores se decantan por los foros que la propia plataforma Chamilo o la red Ning proporcionan.

Creemos que el tipo de elección y la utilización de las redes sociales para el aprendizaje en el MOOC están unidas a una cultura de uso de Internet y sus 
herramientas. Así al igual que señala el estudio anual de redes sociales de 2014 (IAB, 2014), son los jóvenes de 18 a 30 años los que usan más las redes sociales, dato que coincide con los resultados recogidos en esta investigación. Es más, el grupo de menores o igual a 31 años califica la red social Twitter y los blogs como las mejores redes para el aprendizaje. En cambio, ya sea por la falta de familiaridad o por su cultura de aprendizaje, el grupo de mayores de 31 años se decanta por los foros, que son herramientas más cerradas y unidas al contexto del curso y al control del profesorado implicado.

En relación a la utilización de redes sociales es reseñable también que únicamente su uso no conlleva un cambio radical en la forma de aprender, pero, en el caso del MOOC desarrollado, ha facilitado la reflexión sobre el papel que cada estudiante toma en el transcurso del curso online, abierto y masivo. Hemos podido constatar que, además de ser una consideración amplia y muy aceptada que la colaboración e implicación en las actividades interactivas es un factor importante para el aprendizaje personal, el alumnado se comporta de forma más activa y colaboradora de lo que al principio del curso se propone o piensa que va a ser. Se puede considerar, por tanto, que en este caso, ha cambiado su forma de actuar en el MOOC en relación a otro tipo de cursos, por lo que su concepción se traduce, en la realidad del curso, en una colaboración entre iguales. Lo que se puede valorar como un paso adelante teniendo en cuenta las cifras de entradas a foros y blogs, discusiones abiertas o miembros activos en Twitter. Consideramos que el hecho de que los resultados de rendimiento hayan sido favorables y los datos de participación sean altos, pueden estar relacionados con el uso intensivo de diferentes redes sociales que se ha hecho a lo largo del curso. Es más, el darles la posibilidad de elegir la red o las redes que deseaban utilizar ha contribuido a que hubiera mayor interacción y que los resultados globales sean satisfactorios. Es decir, cada tipo de estudiante ha utilizado las redes con las que estaba más familiarizado o que cubría mejor sus necesidades de aprendizaje y esto ha sido valorado positivamente por los propios estudiantes. Puede concluirse, por tanto, que la utilización de Redes Sociales como estrategia de aprendizaje en un MOOC influye positivamente tanto en el rendimiento académico de los alumnos como en la mejora de los porcentajes de éxito y en las tasas de finalización de los estudiantes.

En conclusión, pensamos que todavía la mayoría de los potenciales participantes de MOOC no están totalmente preparados para el aprendizaje mediante la interacción mutua total y poco dirigida por el profesorado, tal y como defiende Siemens (2014) y se practica en los MOOC. Estamos convencidos de que la colaboración entre iguales y la creación de redes en los cursos MOOC, aunque se vaya haciendo de forma más híbrida y escalonada en cursos online, masivos y abiertos cooperativos, nos abre nuevos horizontes de desarrollo para aprender y compartir conocimiento en la enseñanza superior universitaria. 


\section{Referencias bibliográficas}

ALARIO-HOYOS, C., PÉREZ-SANAGUSTÍN, M., DELGADO-KLOOS, C., PARADA, H. A., MUÑOZ-ORGANERO, M., Y RODRÍGUEZ-DE-LAS-HERAS, A. (2013). Analysing the impact of built-in and external social tools in a MOOC on educational technologies. In D. Hernández-Leo, T. Ley, R. Klamma, \& A. Harrer (Eds.), Scaling up learning for sustained impact, 5-18. Berlin, Germany: Springer Berlin Heidelberg. (DOI: 10.1007/978-3-642-40814-4_2).

CABERO, J., LLORENTE, M.C. Y VÁZQUEZ, A.I. (2014). Las tipologías de MOOC: su diseño e implicaciones educativas. Revista de Currículum y Formación de Profesorado, 18 (1), 13-26.

CASTAÑO, C. Y CABERO, J. (Coords.) (2013). Enseñar y aprender en entornos mlearning. Madrid: Síntesis.

CHENG, J.C.Y. (2014). An Exploratory Study of Emotional Affordance of a Massive Open Online Course. European Journal of Open, Distance and e-Learning 17 (1), 43-55.

CONOLE, G. (2013). Los MOOC como tecnologías disruptivas: estrategias para mejorar la experiencia de aprendizaje y la calidad de los MOOCs. Campus Virtuales, 2 (2), 16-28.

FIDALGO, A., SEIN-ECHALUCE, M ${ }^{\mathrm{a}}$ L. Y GARCÍA-PEÑALVO, F. J. (2013). MOOC cooperativo. Una integración entre cMOOC y xMOOC”. In Á. Fidalgo, M.L. Sein-Echaluce (Eds.). Actas del II Congreso Internacional sobre Aprendizaje, Innovación y Competitividad, CINAIC, 481-486. Madrid: Fundación General de la Universidad Politécnica de Madrid.

KARSENTI, T. (2013). The MOOC. What the research says. International Journal of Technologies in Higher Education, 10 (2), 23-37.

KHALIL, H. Y EBNER, M. (2013). How satisfied are you with your MOOC?. A research study of interaction in huge online courses. In Herrington, J. et al. (Eds), Proocedings of World Conference on Educational Multimedia, Hypermedia and Telecommunications 2013, 830-839. Chesapeake, Virginia: Association for the Advancement of Computing in Education (AACE).

KOP, R., FOURNIER, H. \& MAK, S.F.J. (2011). A Pedagogy of Abundance or a Pedagogy to Support Human Beings? Participant support on Massive Open Online Courses. International Review of Research in Open and Distance Learning, 12 (7), 74-93.

LIYANAGUNAWARDENA, T.R. (2014). MOOC experience: a participant's reflection. ACM SIGCAS Computers and Society, 44 (1), 9-14. (DOI)10.1145/2602147.2602149.

LIYANAGUNAWARDENA, T.R., ADAMS, A.A. Y WILLIAMS, S.A. (2013). MOOCs: A Systematic Study of the Published Literature 2008-2012. The 
International Review of Research in Open and Distance Education, 14, (3), 202227.

RODRIGUEZ, C.O. (2012). MOOCs and de AI-Stanford like courses: Two Successful and Distinct Course Formats for Massive Open Online Courses. European Journal of Open, Distance and E-Learning, 2012 (II), 1-13.

RODRIGUEZ, C.O. (2013). The concept of openness behind c and x-moocs (massive open online courses). Open Praxis, 5 (1), 67-73.

SAADATMAND, M. Y KUMPULAINEN, K. (2014). Participants' Perceptions of Learning and Networking in Connectivist MOOCs. MERLOT Journal of Online Learning and Teaching , 10 (1), 16-30.

VALVERDE, J. (2014). MOOCs: una visión crítica desde las Ciencias de la Educación. Revista de Currículum y Formación de Profesorado, 18 (1), 93-111.

WOJCIECHOWSKI, R. Y CELLARY, W. (2013). Evaluation of learners'attitude toward learning in ARIES augmented reality environments. Computers \& Education, 68, 570-585 [formato html] DOI>10.1016/j.compedu.2013.02.014 (Consultado 1 de julio de 2014).

YEAGER, C., HURLEY-DASGUPTA, B., Y BLISS, C. A. (2013). MOOCs and global learning: An authentic alternative. Journal of Asynchronous Learning Networks, 17 (2), 133-147.

\section{Referencias digitales}

ÁLVAREZ, D. (19 setiembre 2013). Éxito y fracaso en un MOOC, Algunas reflexiones [formato html] http://e-aprendizaje.es/2013/09/19/exito-y-fracaso-enun-mooc/ (Consultado 15 de mayo de 2014).

BATES, T. (5 agosto 2012). What's right and what's wrong about Coursera-style MOOCs [formato html] http://www.tonybates.ca/2012/08/05/whats-right-andwhats-wrong-about-coursera-style-moocs (Consultado 15 de febrero de 2014).

CASTAÑO, C. (9 julio 2013). Tendencias en la investigación en MOOCs. Primeros resultados [formato PDF] http://ikasnabar.com/papers/castano1 (Consultado 10 de febrero de 2014).

CONOLE, G. (15 enero 2014). Reviewing the trajectories of e-learning [formato html] http://e4innovation.com/?p=791 (Consultado 8 de febrero de 2014).

EYNON, R. (2014). Conceptualising interaction and learning in MOCs. MOOC Research Initiative Final Report [formato PDF] http://www.moocresearch.com/wpcontent/uploads/2014/06/C9146_EYNON_MOOC-Research-

InitiativeEynon9146Final.pdf (Consultado 30 de abril de 2014).

GATES FOUNDATION (s/f). MOOC Research Initiative [formato html] http://www.moocresearch.com/ (Consultado 13 de diciembre de 2013). 
GUÀRDIA, L., MAINA, M. \& SANGRÀ, A. (9 mayo 2013). MOOC design principles: A pedagogical approach from the learner's perspective . eLearning papers, $\quad 33, \quad 1-6 . \quad$ [formato $\quad h t m l]$ http://www.openeducationeuropa.eu/en/article/MOOC-Design-Principles.-APedagogical-Approach-from-the-Learner\%E2\%80\%99s-Perspective (Consultado 1 de julio de 2014).

HAGGARD, S. (2013). The maturing of the MOOC. BIS research paper number 130: literature review of massive online courses and other forms of online learning. Londres: Department for Bussines Innovation \& Skills. [formato PDF] https://www.gov.uk/government/uploads/system/uploads/attachment_data/file/2401 93/13-1173-maturing-of-the-mooc.pdf (Consultado 30 de abril de 2014).

IAB SPAIN (2014). V Estudio anual de redes sociales. Interactive Advertising Bureau (IAB) [formato PDF] http://www.iabspain.net/wpcontent/uploads/downloads/2014/04/V-Estudio-Anual-de-Redes-Socialesversi\%C3\%B3n-reducida.pdf (Consultado 30 de abril de 2014).

JOHNSON, L., ADAMS BECKER, S., CUMMINS, M., ESTRADA, V., FREEMAN, A.Y LUDGATE, H. (2013). NMC Horizon Report. 2013 Higher Education Edition. Austin, Texas: The New Media Consortium. [formato PDF] http://www.nmc.org/pdf/2013-horizon-report-HE.pdf (Consultado 30 de abril de 2014).

JOHNSON, L., ADAMS, S. Y CUMMINS, M. (2012). NMC Horizon Report. 2012 Higher Education Edition. Austin, Texas: The New Media Consortium. [formato PDF] http://www.nmc.org/pdf/2012-horizon-report-HE.pdf (Consultado 30 de abril de 2014).

JORDAN, K. (2013). MOOC completion rates: The data [formato html] http://www.katyjordan.com/MOOCproject.html (Consultado 15 de diciembre de 2013).

KNOX, J., BAYNE, S., MACLEOD, H., ROSS, J. Y SINCLAIR, C. (8 agosto 2012). MOOC Pedagogy: the challanges of developing for coursera [formato html] https://newsletter.alt.ac.uk/2012/08/mooc-pedagogy-the-challenges-of-developingfor-coursera/ (Consultado 3 de diciembre de 2013).

KOLOWICH, S. (16 junio 2013). The professors behind the MOOC [formato html] http://chronicle.com/article/The-Professors-Behind-the-

MOOC/137905/\#id=overview (Consultado 5 de mayo de 2014).

LANE, L. (15 agosto 2012). Three kinds of MOOCs [formato html] http://lisahistory.net/wordpress/2012/08/three-kinds-of-moocs (Consultado 10 de febrero de 2013).

OLIVER, M., HERNÁNDEZ,-LEO, D., DAZA, V., MARTÍN, C. Y ALBÓ, L. (2014). MOOCs en España. Panorama actual de los Cursos Masivos Abiertos en Línea en las Universidades Españolas. Social Innovation in Education. Barcelona: Cuaderno Red de Cátedras Telefónica. Universitat Pompeu Fabra [formato PDF] 
http://www.catedratelefonica.upf.edu/wp-content/uploads/2014/02/MOOCs-enEspa\%C3\%B1a1.pdf (Consultado 30 de abril de 2014).

OPEN EDUCATION EUROPA (1 julio 2014). European MOOC Scoreboard. [formato html] http://www.openeducationeuropa.eu/es/european_scoreboard_moocs (Consultado 1 de julio de 2014).

POELLHUBER, B., ROY, N. BOUCHOUCHA, I. Y ANDERSON, T. (2014). The Relationship Between the Motivational Profiles, Engagement Profiles and Persistence of MOOC Participants. Final Report [formato PDF] http://www.moocresearch.com/wp-content/uploads/2014/06/MOOC-ResearchInitiativePoelhuber9187v4a.pdf (Consultado 30 de abril de 2014).

SHIRKY, C. (8 julio 2013). MOOCs and economic reality [formato html] http://chronicle.com/blogs/conversation/2013/07/08/moocs-and-economic-reality/ (Consultado 28 de abril de 2014).

SIEMENS, G. (4 junio 2012). What is the theory that underpins "our" MOOCs? [formato html] http://www.elearnspace.org/blog/2012/06/03/what-is-the-theorythat-underpins-our-moocs/ (Consultado 28 de febrero de 2014).

SIEMENS, G. (6 de mayo 2014). Multiple pathways: Blending xMOOCs \& cMOOCs [formato html] http://www.elearnspace.org/blog/2014/05/06/multiple-pathwaysblending-xmoocs-cmoocs/ (Consultado 18 de mayo de 2014).

VARDI, MI (2012). Will MOOCs destroy academia? Communications of the ACM, 55 (11). [formato html] http://dl.acm.org/citation.cfm?id=2366317 (Consultado 24 de junio de 2014).

WANG, Y. Y BAKER, R. (Junio 2014). MOOC Learner Motivation and Course Completion Rates. MOOC Research Initiative. Final Report [formato PDF] http://www.moocresearch.com/wp-content/uploads/2014/06/MRI-ReportWangBaker-June-2014.pdf (Consultado 30 de junio de 2014).

ZAPATA-ROS, M. (2013). MOOC, una visión crítica y una alternativa complementaria: la individualización del aprendizaje y de la ayuda pedagógica. $\begin{array}{llllll}\text { Campus } & \text { Virtuales, } & 1 & \text { (2), 20-38 } & \text { [formato pdf] }\end{array}$ http://www.revistacampusvirtuales.es/campusvirtuales/2/2.pdf (Consultado 30 de junio de 2014). 


\section{Correspondencia con los autores}

Carlos CASTAÑO GARRIDO

Departamento de Didáctica y Organización Escolar

Escuela Universitaria de Magisterio de Bilbao

Barrio Sarriena s/n

48940 Leioa (Bizkaia)

e-mail: carlos.castano@ehu.eus

Inmaculada MAIZ OLAZABALAGA

Departamento de Psicología Evolutiva y de la Educación

Escuela Universitaria de Magisterio de Bilbao

Barrio Sarriena s/n

48940 Leioa (Bizkaia)

e-mail: inmaculada.maiz@ehu.eus

Urtza GARAY RUIZ

Departamento de Didáctica de la Lengua y la Literatura

Escuela Universitaria de Magisterio de Bilbao

Barrio Sarriena s/n

48940 Leioa (Bizkaia)

e-mail: urtza.garay@ehu.eus 